$x$ and $x^{\prime}$ are angular diameters of the first rings; and $p$ and $p^{\prime}$ are the corresponding orders of interference. The problem may be simplified, however, by a variation in the method which requires a measurement of only linear diameters. In the simpler method the above expression is replaced by the more fundamental one, from which indeed it is derived:

$$
\frac{\lambda}{\lambda^{\prime}}=\frac{p^{\prime}+a^{\prime}}{p+a}, .
$$

where $a$ is the fractional part of the number which gives the order of interference for wave-length $\lambda$, corresponding to the centre of the ring system. Now the value of $a$ (and $a^{\prime}$ ) may readily be obtained from measurements of linear diameters by making use of the relation

$$
a=\frac{(n-\mathrm{I}) d_{k}^{2}-(k-\mathrm{I}) d_{n}^{2}}{d_{n}^{2}-d_{k}^{2}},
$$

where $d_{k}$ and $d_{n}$ are linear diameters of the $k$ th and the $n$th rings. This expression, which is a generalised form of one used by Merton (Proc. Roy. Soc. A, 96, 388 , I920) in an investigation on the spectra of isotopes, may be deduced easily from the fundamental equation of the étalon (path difference $=2 t \cos x_{n}$ ). The accompanying table, in which numbers are calculated from measurements of a plate taken by one of the writer's students, gives an idea of the accuracy of the method.

\begin{tabular}{|c|c|c|}
\hline$k$. & $n$. & $\alpha$. \\
\hline 2 & 3 & $0.058 \mathrm{I}$ \\
2 & 4 & $0.058 \mathrm{I}$ \\
2 & 5 & 0.0579 \\
3 & 4 & 0.0584 \\
3 & 5 & 0.0577 \\
4 & 5 & 0.0565 \\
\hline
\end{tabular}

Mean $=0.0578 \pm 0.00007$.

The method may be varied by using equation ( $\mathrm{I}$ ) and calculating the value of $f$, the focal length which determines the angular diameter of a ring from the relation

$$
p=\frac{8 f^{2}(n-k)}{{d n_{n}^{2}}^{2}-d_{k^{2}}^{2}}+k-\mathrm{r} .
$$

There is, of course, little, if any, advantage in following this procedure.

JoHn K. RoBERTSON.

Queen's University, Kingston, Canada, May 20.

\section{On the Centroid of a Circular Arc.}

THE usual text-book formula for the radial distance of the centroid of a circular arc of radius $r$ and length 2 ra, may be converted into a useful result by expressing the position of the centroid in terms of its distance from the chord of the arc and as a fraction of the versine of the arc. Thus the usual form

$$
\stackrel{\bar{v}}{r}=\frac{\sin \alpha}{a}
$$

converts to

$$
\bar{h}=\frac{\frac{\sin \alpha}{a}-\cos a}{\mathrm{r}-\cos \alpha} .
$$

If this is expanded in powers of $a$ we have

$$
\begin{aligned}
& \bar{h}=\frac{1}{3}-a^{2}(\mathrm{I} / 30)+a^{4}(\mathrm{I} / 840)- \\
& \frac{1}{2}-a^{2}(\mathrm{I} / 24)+a^{4}(\mathrm{I} / 720)- \\
&=\frac{2}{3}\left(\mathrm{I}-\frac{a^{2}}{60}+\frac{a^{4}}{1260}-. . .\right) .
\end{aligned}
$$

Thus for flat arcs the centre of gravity is at $\frac{2}{3}$ rds of the vertex of the arc, and for an arc of 2 radians the error is only I.5 per cent. Even with a semicircle this value is in error by only about 6 per cent. This result is the more interesting, because no simple curve except the straight line has its centre of gravity at a height proportional to the terminal ordinate.

H. S. ROWELL,

Director of Research, Research Association of British Motor and Allied Manufacturers.

I 5 Bolton Road,

Chiswick, W.4, May 26.

\section{Einstein and Mach.}

IN the issue of NATURE for August I8, I923, p. 253, Prof. Einstein is quoted as having said of Mach, at the reception given to him (Einstein) by the Société Française de Physique, "His (Mach's) view of science, that it deals with immediate data, led him to reject the existence of atoms. Probably were he still with us he would change his opinion." As one of the oldest pupils of Mach who worked with him on fluorescence in 1877 and enjoyed his warm friendship since 1876 , I often had an opportunity of discussing atomism with him. Not long before the War, I was sitting with him in his garden in Vienna, and I remember that he said to me: "Atomism is a good working hypothesis for the study of chemistry; it must be used with great care on studying and working in science; but it is extremely dangerous as a noetic theory." It was after the memorable discovery of Rutherford and Geiger in 1908, who showed that the emission of $a$-particles from radium-C is the same whether it be counted by the spinthariscope or by the electroscope, and the most incredulous chemists admitted it as a proof of the existence of atoms, that Mach did not change his old view. At the beginning of the War, I asked him in a letter whether he considered the results of all the observations in radioactivity as a proof of the existence of atoms, and he replied to me verbally: "I do not make myself a proselyte of my ideas-do not make yourself a proselyte on atomism." $\mathrm{He}$ never changed his ideas up to his death.

Bohuslav Brauner.

Chemical Institute, University, Prague VI.

\section{Approximate Integration.}

THE following rule for approximate integration generally gives almost as good results with four, seven, or ten ordinates as Simpson's does with seven, eleven, and fifteen respectively.

$$
\int y d x=h\left\{2\left(y_{2}+y_{6}+y_{10}+\ldots\right)+\left(y_{3}+y_{5}+y_{7}+\ldots\right)\right\} \text {. }
$$

The rule is obtained by applying the trapezoidal rule to the ordinates $y_{1}, 2 y_{2}-\frac{1}{2}\left(y_{1}+y_{3}\right), y_{3}, \frac{1}{2}\left(y_{3}+y_{5}\right)$, $y_{5}, 2 y_{6}-\frac{1}{2}\left(y_{5}+y_{7}\right), y_{7}$, etc.

Taking six spaces $\int_{0}^{1} y d x=\frac{1}{3}\left(y_{2}+y_{6}\right)+\frac{1}{6}\left(y_{3}+y_{5}\right)$.

When applied to the areas mentioned by $M$. Fréchet in his letter in Nature of May I7, the rule gives the following errors for $\int_{0}^{1} y d x$ :

$$
\begin{array}{r}
y, \sqrt{ } x-x^{2}, \sqrt{ } \mathrm{I}-x^{2}, \sqrt{ } 4 x-x^{2}, \log (\mathrm{I}+x), \quad e^{x}, \mathrm{I} / \mathrm{I}+x, \\
\mathrm{O} .0028,0.004 \mathrm{I}, \quad 0.0 \mathrm{I} 2, \quad 0.0003,0.0002,0.0004, \\
2 / \mathrm{I}+x, \sin x, y \mathrm{I} / \mathrm{I}+25 x^{2} . \\
0.0000,0.012, \quad 0.012 .
\end{array}
$$

These results are obtained by using four ordinates, and they compare well with those calculated by Simpson's rule using seven ordinates, but the advantage of this rule is still greater when more ordinates are used. H. V. LOWRY.

NO. 2852 , VOL. I I 3$]$ 